\title{
The Superconducting Instabilities of the non half-filled Hubbard Model in Two Dimensions
}

\author{
D. Zanchi and H. J. Schulz \\ Laboratoire de Physique des Solides, Université Paris-Sud, 91405 Orsay, France
}

\begin{abstract}
The problem of weakly correlated electrons on a square lattice is formulated in terms of one-loop renormalization group. Starting from the action for the entire Brillouin zone (and not with a low-energy effective action) we reduce successively the cutoff $\Lambda$ about the Fermi surface and follow the renormalization of the coupling $U$ as a function of three energy-momenta. We calculate the intrinsic scale $T_{c o}$ where the renormalization group flow crosses over from the regime $\left(\Lambda>T_{c o}\right)$ where the electron-electron (e-e) and electron-hole (eh) terms are equally important to the regime $\left(\Lambda<T_{c o}\right)$ where only the e-e term plays a role. In the low energy regime only the pairing interaction $V$ is marginally relevant, containing contributions from all renormalization group steps of the regime $\Lambda>T_{c o}$. After diagonalization of $V_{\Lambda=T_{c o}}$, we identify its most attractive eigenvalue $\lambda_{\min }$. At low filling, $\lambda_{\min }$ corresponds to the $B_{2}$ representation $\left(d_{x y}\right.$ symmetry), while near half filling the strongest attraction occurs in the $B_{1}$ representation $\left(d_{x^{2}-y^{2}}\right.$ symmetry). In the direction of the van Hove singularities, the order parameter shows peaks with increasing strength as one approaches half filling. Using the form of pairing and the structure of the renormalization group equations in the low energy regime, we give our interpretation of ARPES experiments trying to determine the symmetry of the order parameter in the Bi2212 high- $T_{c}$ compound.
\end{abstract}


PACS numbers: 74.10, 74.20, 75.10L

Typeset using REVTEX 


\section{INTRODUCTION}

An immense number of recent experiments on high $T_{c}$ superconductors aims at a determination of the form of the BCS gap function in momentum space. 1 Josephson junction

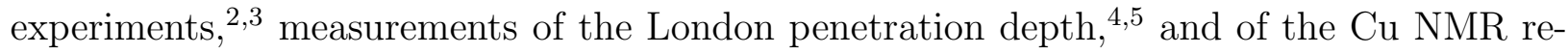

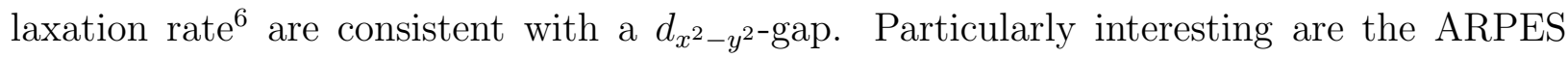
data which provide rather precise information about the detailed angular dependence of the amplitude of the gap function. The experiments on the Bi2212 compound show that the order parameter is maximal along the $(0, \pi)$ direction 7 目 and that its amplitude in the $(\pi, \pi)$ direction seems to attain a nonzero value at a new critical temperature below $T_{c}$.

The $d$ symmetry of the gap function is generally considered to be a sign of a pairing interaction of electronic origin, implying the absence of the standard phononic mechanism for superconductivity. The idea of a superconducting state induced by fluctuations of purely electronic origin in systems of electrons with Coulomb repulsion is originally due to Kohn and Luttinger 10 for the case of the three dimensional electron gas. Similar effects exists in a two-dimensional electron gas, and generally they depend strongly on the form of the Fermi surface. Perturbative calculations of the four point vertex for a weakly filled band in the Hubbard model show that the model is instable against $d_{x y}$ superconductivity 11. Quantum Monte Carlo calculations on the same model, in the vicinity of metal-insulator transition, show that the attractive pairing interaction of $d_{x^{2}-y^{2}}$ symmetry is dominant, 12 , in agreement with the earlier arguments that antiferromagnetic fluctuations are the mediator of pairing interactions 13 - Direct evidence for $d_{x^{2}-y^{2}}$ superconducting ordering has however not yet been found in quantum Monte Carlo studies. Antiferromagnetic fluctuations become stronger and stronger as one approaches half-filling. For weakly interacting electrons, these fluctuations are associated with the $\ln ^{2} T$-divergence of the electron-hole (e-h) loop diagram, caused by the nesting property of the Fermi surface and the van Hove singularities. On the other hand the BCS fluctuations, characterized by the electron-electron (e-e) loop, normally only linear in logarithms, crosses over to a $\ln ^{2} T$ form in the vicinity of half-filling. The 
perturbative treatment of an interacting system of electrons should thus be based on the summation of all iterations of these two types of loops. The renormalization group is one way to do this. Applied to interactions between electrons placed at the van Hove points it gives an antiferromagnetic instability at half-filling and superconductivity of $d_{x^{2}-y^{2}}$ symmetry if the deviation of the chemical potential $\mu$ from its value at half-filling becomes of the order of critical temperature of the antiferromagnetic state.20 The equivalent parquet approach has been used for half-filling and also finds the antiferromagnetic state.21,22 A direct calculation of the zero temperature free energy 23 up to the second order in the bare interaction $U_{0}$ confirms that at half-filling the antiferromagnetic order is stable, but finds no finite superconducting order parameter at any filling.

In the present analysis we search to know whether the Hubbard model with repulsive on-site interaction can lead to superconductivity, and if it does, to what form of the gap function. In particular, we are interested in the dependence of the results on the density of electrons. It is hoped that the results can help us to clarify the origins of the existence of a highly anisotropic BCS gap function in the cuprates.

The renormalization group technique for fermionic systems in two and three dimensions has recently been developed, but with very drastic limitations. The Wilsonian modeelimination technique was applied by Shankar24 only to systems with an either isotropic or open, perfectly nested Fermi surface. Weinberg25 has written the flow equations for a general case of an anisotropic Fermi surface, but taking into account only the electron-electron channel of the flow. Moreover, a common tendency is to do the renormalization group procedure only for a thin ring of degrees of freedom around the Fermi surface and to linearize the spectrum in the radial direction, taking as a starting model the low-energy effective action. This makes it difficult or impossible to make statements about the phase diagram of lattice models like those relevant for the description of the cuprates, due to the absence of a proper description of high-energy degrees of freedom, the elimination of which may considerably affect the effective low-energy action.

We formulate the renormalization group starting from the Hubbard model, with the 
whole Brillouin zone involved in renormalization. We do no tree level scaling before the energy shell $\pm \Lambda$ of available states around the Fermi surface contains no van Hove singularity. Once $\Lambda$ has become the smallest energy scale, performing a tree level analysis and deriving the renormalization group equations, we show that the effective coupling function contains some relevant contributions with the origins in the regime with higher $\Lambda$, i.e. from the electronic degrees of freedom not included in the low energy effective action. We must add that our renormalization group, since perturbative in interaction, can provide uniquely the information whether the Fermi liquid is a fixed point or not. If the coupling flows to strong coupling, we can say in which direction it flows, for example in the $d$-type superconducting direction, but we can not say whether another fixed point with finite superconducting order parameter with $d$ symmetry exists or not. This kind of problem is well known e.g. from the renormalization group in quasi-one-dimensional compounds, where the most divergent flow in some direction is always associated with the corresponding long-range order (LRO) because already infinitesimal interchain coupling suffices for its stabilization.26 Similarly, the dimensionality reason for the non-existence of LRO in two dimensions at finite temperature can be ignored as soon as small hopping in the third direction exists. This, however, doesn't mean that LRO and Fermi liquid fixed points are the only possible, on this question the one-loop renormalization group simply can not give an answer.

The problem is formulated in section 2 in terms of the effective action and the corresponding renormalization group flow equation for the coupling function. In section 3 , after calculation of the crossover energy to the purely electron-electron (e-e) part of the flow, we derive the renormalization group equation for the pairing function $V$. In section 4 . we diagonalize the pairing $V$ for the case of a non-divergent electron-hole (e-h) channel and determine the most attractive eigenfunction and the resulting critical temperature as functions of the chemical potential $\mu$. In section 5 we give our picture of the gap viewed by ARPES experiments. The conclusions are given in section 6 . 


\section{MODEL AND FORMULATION OF THE RENORMALIZATION GROUP THEORY}

The Hubbard model for a two-dimensional system of electrons on a square lattice is given by

$$
H=\sum_{\sigma \mathbf{k}} \xi_{\mathbf{k}}^{0} a_{\sigma \mathbf{k}}^{\dagger} a_{\sigma \mathbf{k}}+\frac{1}{2} U_{0} \sum_{\sigma} \sum_{\mathbf{k}_{1}, \mathbf{k}_{2}, \mathbf{k}_{3}} a_{-\sigma, \mathbf{k}_{1}+\mathbf{k}_{2}-\mathbf{k}_{3}}^{\dagger} a_{-\sigma \mathbf{k}_{2}} a_{\sigma \mathbf{k}_{3}}^{\dagger} a_{\sigma \mathbf{k}_{1}},
$$

where $\xi_{\mathbf{k}}^{0}=-2 t\left(\cos k_{x}+\cos k_{y}\right)-\mu$ and the momenta are within the first Brillouin zone. For this model we introduce now the action in terms of fermion coherent states represented by Grassmann variables $27 \Psi_{\sigma K}$ and $\bar{\Psi}_{\sigma K}$, where $K=(\mathbf{k}, \omega)$. We will write it in the form

$$
\begin{array}{r}
S\left\{\Lambda_{0}, \xi_{\mathbf{k}}^{0}, U_{0}\right\}=\int_{0}^{\infty} \frac{d \tau}{2 \pi} \sum_{\sigma \mathbf{k}} \Theta\left(\Lambda_{0}-\left|\xi_{\mathbf{k}}^{0}\right|\right) \bar{\Psi}_{\sigma K}\left(\partial_{\tau}-\xi_{\mathbf{k}}^{0}\right) \Psi_{\sigma K}+ \\
+\frac{1}{2} \sum_{\sigma \sigma^{\prime}} \int\left(\prod_{i=1}^{3} \frac{d \omega_{i}}{2 \pi}\right)_{\mathbf{k}_{\mathbf{1}}, \mathbf{k}_{\mathbf{2}}, \mathbf{k}_{3}} U_{0} \Theta_{\mathbf{k}_{\mathbf{1}}, \mathbf{k}_{\mathbf{2}}, \mathbf{k}_{\mathbf{3}}, \mathbf{k}_{\mathbf{4}}}^{\left(\Lambda_{0}\right)} \bar{\Psi}_{\sigma K_{3}} \bar{\Psi}_{\sigma^{\prime} K_{4}} \Psi_{\sigma^{\prime} K_{2}} \Psi_{\sigma K_{1}}
\end{array}
$$

where $\Theta_{\mathbf{k}_{\mathbf{1}}, \mathbf{k}_{\mathbf{2}}, \mathbf{k}_{\mathbf{3}}, \mathbf{k}_{\mathbf{4}}}^{(\Lambda)} \equiv \prod_{i=1}^{4} \Theta\left(\Lambda-\left|\xi_{\mathbf{k}_{\mathbf{i}}}^{0}\right|\right)$ constrains all four momenta to run within the energy shell $\pm \Lambda_{0}=8 t$ around the Fermi surface. The energy and momentum are conserved so that $K_{4}\left(K_{1}, K_{2}, K_{3}\right)=\left(\omega_{1}+\omega_{2}-\omega_{3}, \mathbf{k}_{\mathbf{1}}+\mathbf{k}_{\mathbf{2}}-\mathbf{k}_{\mathbf{3}}\right)$. Note that the size of the cutoff is equal to the bandwidth, i.e. the whole Brillouin zone is available for integration. Thus, the $\Theta$ functions have no meaning yet: they become important when the cutoff, reduced by the renormalization group, become lower than a tilt from the Fermi level to the band boundary. Note that for a non-half filled band the effective phase space is not particle-hole symmetric.

The renormalization group transformation that we will use, known as the field theory approach, is defined as the mapping

$$
S\left\{\Lambda_{0}, \xi_{\mathbf{k}}^{0}, U_{0}\right\} \rightarrow S^{\prime}=S\left\{\Lambda_{0} \rightarrow \Lambda_{0} e^{-l}, \xi_{\mathbf{k}}^{0} \rightarrow \xi_{\mathbf{k}}, U_{0} \rightarrow U\left(K_{1}, K_{2}, K_{3}\right)\right\}
$$

where $\xi_{\mathbf{k}}$ and $U$ depend on $l$ in a way so that the physical properties of $S^{\prime}$ and $S$ are the same for energies lower than $\Lambda=\Lambda_{0} e^{-l}$. This requirement is fulfilled if all one-particle irreducible vertices are invariant under reduction of the cutoff from $\Lambda_{0}$ to $\Lambda_{0} e^{-l}$. The renormalization group can be thought of as a set of successive, infinitesimally small steps $d l$. This allows 
us to formalize the renormalization group requirement in a set of equations $\partial_{l} \Gamma_{i}=0$, where $i=2,4,6, \ldots$ Up to second order in $U$, it suffices to consider only $\Gamma_{2}$ and $\Gamma_{4}$, because all higher vertices are of higher order in $U$. The solutions of the equations $\partial_{l} \Gamma_{2}=0$ and $\partial_{l} \Gamma_{2}=0$ give us the renormalization group flow for $\xi_{\mathbf{k}}$ and $U\left(K_{1}, K_{2}, K_{3}\right)$.

Conservation of spin allows us to write the interaction part of the action as a sum of the singlet $\left(\left|\vec{\sigma}+\overrightarrow{\sigma^{\prime}}\right|=0\right)$ and triplet $\left(\left|\vec{\sigma}+\overrightarrow{\sigma^{\prime}}\right|=\sqrt{2}\right)$ parts:

$$
\bar{s}\left(K_{4}, K_{3}\right) U^{S}\left(K_{1}, K_{2}, K_{3}\right) s\left(K_{2}, K_{1}\right)+\bar{t}_{\mu}\left(K_{4}, K_{3}\right) U^{A}\left(K_{1}, K_{2}, K_{3}\right) t_{\mu}\left(K_{2}, K_{1}\right)
$$

where $s$ and $t_{\mu}$ are the variables of annihilation of the singlet and triplet states

$$
\begin{aligned}
& s\left(K_{2}, K_{1}\right) \equiv \frac{1}{\sqrt{2}} \sum_{\sigma} \sigma \Psi_{\sigma K_{2}} \Psi_{-\sigma K_{1}}, \\
& t_{0}\left(K_{2}, K_{1}\right) \equiv \frac{1}{\sqrt{2}} \sum_{\sigma} \Psi_{\sigma K_{2}} \Psi_{-\sigma K_{1}} \quad ; \quad t_{ \pm 1}\left(K_{2}, K_{1}\right) \equiv \Psi_{\uparrow, \downarrow K_{2}} \Psi_{\uparrow, \downarrow K_{1}} .
\end{aligned}
$$

The singlet state is symmetric and the triplet antisymmetric under exchange of the momenta of two particles. Correspondingly, the coupling function $U^{S}\left(K_{1}, K_{2}, K_{3}\right)$ can be taken to be symmetric and $U^{A}\left(K_{1}, K_{2}, K_{3}\right)$ to be antisymmetric under the momentum exchange operation $X$, defined as

$$
X \mathcal{F}\left(K_{1}, K_{2}, K_{3}\right)=\mathcal{F}\left(K_{2}, K_{1}, K_{3}\right)
$$

$\mathcal{F}$ being a function of four energy-momenta which conserves energy and momentum. If $\mathcal{F}$ possesses time-reversal symmetry

$$
\mathcal{F}\left(K_{1}, K_{2}, K_{3}\right)=\mathcal{T} \mathcal{F}\left(K_{1}, K_{2}, K_{3}\right) \equiv \mathcal{F}\left(K_{3}, K_{4}\left(K_{1}, K_{2}, K_{3}\right), K_{1}\right)
$$

which certainly is a property of the vertex, then it is equivalent whether $X$ exchanges $K_{1}$ and $K_{2}$ or $K_{3}$ and $K_{4}$, i.e. $\mathcal{F}\left(K_{2}, K_{1}, K_{3}\right)=\mathcal{F}\left(K_{1}, K_{2}, K_{4}\left(K_{1}, K_{2}, K_{3}\right)\right)$. Formally, $U^{S}$ and $U^{A}$ are given by

$$
U^{A}=\frac{1}{2}(1-X) U, \quad U^{S}=\frac{1}{2}(1+X) U
$$

On the other hand, the interaction can also be written as a sum of one term with equal $\left(\sigma=\sigma^{\prime}\right)$ and one with opposite $\left(\sigma=-\sigma^{\prime}\right)$ spin quantum numbers, with corresponding 
coupling functions named $U_{\|}\left(K_{1}, K_{2}, K_{3}\right)$ and $U_{\perp}\left(K_{1}, K_{2}, K_{3}\right)$, respectively. From two equalspin electrons one can build only a triplet state, which make us conclude that

$$
U_{\|}=U^{A}
$$

while

$$
U_{\perp}=U=U^{A}+U^{S}
$$

containing the singlet and the triplet interactions.

Another way to write the interaction is in terms of a charge and a spin part

$$
U_{c}\left(K_{1}, K_{2}, K_{3}\right) \bar{C}\left(K_{2}, K_{4}\right) C\left(K_{3}, K_{1}\right)+U_{\sigma}\left(K_{1}, K_{2}, K_{3}\right) \overline{\mathbf{S}}\left(K_{2}, K_{4}\right) \cdot \mathbf{S}\left(K_{3}, K_{1}\right),
$$

where $C$ and $S_{i}$ are

$$
C\left(K_{3}, K_{1}\right) \equiv \sum_{\sigma} \bar{\Psi}_{\sigma K_{3}} \Psi_{\sigma K_{1}} \quad ; \quad S_{i}\left(K_{3}, K_{1}\right)=\sum_{\sigma \sigma^{\prime}} \bar{\Psi}_{\sigma K_{3}} \sigma_{\sigma \sigma^{\prime}}^{i} \Psi_{\sigma^{\prime} K_{1}}
$$

The charge and spin coupling functions are

$$
U_{c}=\frac{1}{4}(2-X) U, \quad U_{\sigma}=-\frac{X}{4} U .
$$

In first order of perturbation theory, these functions determine the renormalization of the charge-charge and the spin-spin correlation functions.

We proceed now with the derivation of renormalization group equations. For simplicity, we will ignore the renormalization flow of $\xi_{\mathbf{k}}$, which follows from conservation of $\Gamma_{2}$, renormalizing the form of the Fermi surface, the effective mass, etc. This approximation is justified in the case of the circular Fermi surface 2 . In the anisotropic case, the diagrams for $\Gamma_{2}$ have a dependence on the direction of $\mathbf{k}$. Moreover, even a small renormalization of the Fermi energy can give important changes of the form of the Fermi surface if one is close to half-filling, because of van Hove singularities. For filling not too close to one-half we can expect that the essential of the physics is given by just the renormalization of the coupling $U$ using the bare dispersion relation $\xi_{\mathbf{k}}^{0}$, which we will call $\xi_{\mathbf{k}}$ from now on. 
The Feynman diagrams for $\Gamma_{2}$ and $\Gamma_{4 \perp}=\Gamma_{4}$ are given in Fig. 1. The first loop in the expression for $\Gamma_{4}$ is of the electron-electron (e-e) and all others of the electron-hole (e-h) type. Making use of the relations (2.10), (2.11), and (2.9), we get the expression for $\Gamma_{4}$ in terms of $U$ and $X U$. If we write the integration measure of the loop diagrams in the form

$$
\int \frac{d \omega}{2 \pi} \int_{-\Lambda}^{+\Lambda} d \xi \oint \frac{d s}{v(s, \xi)}
$$

$s$ being the curves of constant energy $\xi$, then $d \Gamma_{4}$ corresponds to the integration of the two energy shells of width $|\Lambda| d l$ at $\xi= \pm \Lambda$. We obtain the following flow equation

$$
\frac{\partial U}{\partial l}=\beta_{e e}\{U, U\}+\tilde{\beta}_{e h}\{U, U\}
$$

with

$$
\tilde{\beta}_{e h}\{U, U\}=2 \beta_{e h}\{U, U\}-\beta_{e h}\{U, X U\}-\beta_{e h}\{X U, U\}-X \beta_{e h}\{X U, X U\}
$$

The functionals $\beta_{e e}\left\{U_{1}, U_{2}\right\}$ and $\beta_{e h}\left\{U_{1}, U_{2}\right\}$ are the partial derivatives with respect to $l$ of the e-e and e-h loops and both are bilinear forms in $U_{1}\left(K_{1}, K_{2}, K_{3}\right)$ and $U_{2}\left(K_{1}, K_{2}, K_{3}\right)$. They read

$$
\beta_{e e}\left\{U_{1}, U_{2}\right\}=\left(\Xi\left\{U_{1}, U_{2}\right\}+\Xi\left\{X U_{1}, X U_{2}\right\}\right) \frac{1+\kappa_{e e}}{2}
$$

and

$$
\beta_{e h}\left\{U_{1}, U_{2}\right\}=\left(\Pi\left\{U_{1}, U_{2}\right\}+\mathcal{T} \Pi\left\{U_{1}, U_{2}\right\}\right) \frac{1+\kappa_{e h}}{2}
$$

with

$$
\begin{aligned}
& \Xi\left\{U_{1}, U_{2}\right\}=\frac{-\Lambda}{(2 \pi)^{2}} \sum_{\nu=+,-} \int \frac{d s_{\nu}}{v_{\nu}} \Theta\left(\Lambda-\left|\xi_{\mathbf{k}_{\nu}-\mathbf{q}_{e e}}\right|\right) \int_{-\infty}^{+\infty} \frac{d \omega}{2 \pi} \frac{1}{i \omega-\nu \Lambda} \frac{1}{i\left(-\omega+\omega_{e e}\right)-\xi_{\mathbf{k}_{\nu}-\mathbf{q}_{e e}}} \times \\
& \quad \times U_{1}\left(K_{1}, K_{2}, K_{(\nu)}\right) U_{2}\left(K_{3}, K_{4}, K_{(\nu)}\right), \\
& \Pi\left\{U_{1}, U_{2}\right\}=\frac{-\Lambda}{(2 \pi)^{2}} \sum_{\nu=+,-} \int \frac{d s_{\nu}}{v_{\nu}} \Theta\left(\Lambda-\left|\xi_{\mathbf{k}_{\nu}+\mathbf{q}_{e h}}\right|\right) \int_{-\infty}^{+\infty} \frac{d \omega}{2 \pi} \frac{1}{i \omega-\nu \Lambda} \frac{1}{i\left(\omega+\omega_{e h}\right)-\xi_{\mathbf{k}_{\nu}+\mathbf{q}_{e h}}} \times \\
& \quad \times U_{1}\left(K_{1}, K_{(\nu)}, K_{3}\right) U_{2}\left(K_{4}, K_{(\nu)}, K_{2}\right) .
\end{aligned}
$$


The index $\nu=+,-$ symbolizes two energy shells at $+\Lambda$ and $-\Lambda ; v_{\nu}$ stands for $v\left(s_{\nu}, \xi=\nu \Lambda\right)$; $\omega_{e e} \equiv \omega_{1}+\omega_{2} ; \omega_{e h} \equiv \omega_{1}-\omega_{3} ; \mathbf{q}_{e e} \equiv \mathbf{k}_{1}+\mathbf{k}_{2} ; \mathbf{q}_{e h} \equiv \mathbf{k}_{1}-\mathbf{k}_{3} ; K_{\nu} \equiv\left(\mathbf{k}_{\nu}, \omega\right)$, where $\mathbf{k}_{\nu}$ is the momentum running along the path $s_{\nu} . \kappa_{e e}$ and $\kappa_{e h}$ are non-analytic functions of the momenta, given by

$$
\kappa_{e e}=\left\{\begin{array}{ll}
0 & \text { for } \mathbf{q}_{e e}=0 \\
1 & \text { otherwise }
\end{array} \quad ; \quad \kappa_{e h}= \begin{cases}0 & \text { for } \mathbf{q}_{e h}=( \pm \pi, \pm \pi) \\
1 & \text { otherwise }\end{cases}\right.
$$

Their origin is in the derivatives over $\Lambda$ of the products like

$$
\Theta\left(\Lambda-\left|\xi_{\mathbf{k}+\mathbf{q}}\right|\right) \Theta\left(\Lambda-\left|\xi_{\mathbf{k}}\right|\right)
$$

when $\xi_{\mathrm{k}}=\xi_{\mathrm{k}+\mathbf{q}}$.

\section{THE TEMPERATURE SCALE $T_{C O}$}

A particularity of the renormalization group approach treating e-e and e-h fluctuations in more than one dimension is the absence of self-similarity of the problem. In fact, there is an intrinsic energy scale which is a function of the band filling. It is associated with charge and spin fluctuations coming from the e-h term. We will proceed by estimating the characteristic energy scales which appear in $\beta_{e e}$ and $\beta_{e h}$, when all four particles are at the Fermi surface, with zero energy. If we are exactly at half-filling, it is known that in the limit $\omega \rightarrow 0$ both e-e and e-h loops scale like $l^{2}$, which corresponds to the square-logarithmic divergence in both channels. This gives an explicit $\sim l$ dependence in the $\beta$-functionals. Let us suppose now that the filling is slightly lower than one-half, i.e. that $\mu$ is small and negative. We expect two regimes. One is for $l \lesssim l_{x} \sim \ln |8 t / \mu|$, where the flow is still unaffected by the small changes of the Fermi surface due to nonzero $\mu$ and remains proportional to $l$. In the second regime, where $l \gtrsim l_{x}$, the e-e flow is just a constant (i.e. only a ln-divergence),

while the e-h flow decays exponentially due to disappearance of nesting. Even far from half-filling it is possible to define a crossover $l_{x}$, beyond which the flow in the e-h channel disappears exponentially. We can summarize saying that for any filling, $l_{x}$ is a crossover from 
a regime where both $\mathrm{e}-\mathrm{e}$ and $\mathrm{e}-\mathrm{h}$ loops contribute to a regime where $\beta_{\text {eh }}$ starts to behave like $\beta_{e h} \sim \Lambda^{\eta(\mathbf{q})}$. Here $\eta(\mathbf{q})$ is positive for all values of the momentum transfer $\mathbf{q}=\mathbf{q}_{e h}$.

To estimate the dependence of $l_{x}$ on the filling $\langle n\rangle$, we consider the static limit of the partially integrated e-h loop

$$
P_{e h}(l, \mathbf{q}, \omega=0)=\frac{1}{U_{0}^{2}} \int_{0}^{l} \beta_{e h}\left\{U_{0}, U_{0}\right\}
$$

with the momentum transfer $\mathbf{q}$ equal to $2 \mathbf{k}_{F}$ in the direction $(\pi, \pi)$. Note that the energy integration is performed over $8 t<\xi<8 t \exp (-l)$. The derivative of $P_{e h}(l, \mathbf{q}, \omega=0)$ with respect to $l$ gives the explicit $l$-dependence in the $\beta_{e e}$ functional. Fig. 2 shows $P_{e h}(l)$ and $\partial_{l} P_{e h}(l)$ for two different values of $\mu$. It is reasonable to define $l_{x}$ as the point where $\partial_{l} P_{e h}(l)$ starts to decrease. In the exponential regime the function $\partial_{l} P_{e h}(l)$ decays like $\exp (-l / 2)$ (i.e. $\eta\left(2 \mathbf{k}_{F}\right)=1 / 2$, valid for any orientation of $\mathbf{k}_{F}$ ), while the regime $l<l_{x}$ remembers the $\ln ^{2}$ divergence of $P_{e h}$ at half filling. If we consider $P_{e h}(l)$ for some large momentum transfer different from $2 \mathbf{k}_{F}$ (giving intersection rather than touching of the initial- and final-state Fermi surface) we get a shape like $\exp (-l)\left(\right.$ i.e. $\left.\eta\left(\mathbf{q} \neq 2 \mathbf{k}_{F}\right)=1\right)$. The dependence $l_{x}(\langle n\rangle)$ is shown in Fig.3. Near $\langle n\rangle=1$ there is a divergence of the form $l_{x}(\langle n\rangle) \approx \ln |8 t / \mu(\langle n\rangle)|$ because of nesting, while the increasing $l_{x}$ as the filling goes to zero mirrors the fact that, for low density, the Fermi energy appears as the new scale instead of the band-width being used. The insert shows the function $\langle n\rangle(\mu)$.

Once in the exponential regime, $\beta_{e h}$ can be neglected after it becomes smaller than $e^{-1}$ of its value at $l=l_{x}$. Putting $\eta=1 / 2$, this defines the crossover

$$
l_{c o}(\mu)=l_{x}(\mu)+2,
$$

corresponding to the crossover temperature $T_{c o}=8 t \exp \left(-l_{c o}\right)$. Suppose that we now integrate the flow equation (2.16) from $l=0$ to $l=l_{c o}$. Once $l$ has reached $l_{c o}$, only the term $\beta_{e e}\{U\}$ remains in the flow equations, and one has a partially renormalized $U\left(l=l_{c o}\right)$ as initial condition. Now we use the fact that $T_{c o} / 8 t$ is a small parameter, i.e. the inequality $\left|\xi_{\mathbf{k}}\right|<T_{c o}$ determines a thin ring of degrees of freedom, containing no van Hove points, as 
one can conclude looking at Fig.3. This allows us to rescale the momenta $k_{\perp}=\hat{\mathbf{n}}\left(\mathbf{k}-\mathbf{k}_{\mathbf{F}}\right)$, where $\hat{\mathbf{n}}$ is the unit vector normal to Fermi surface, dependent on direction of $\mathbf{k}$. To clarify the reason for which a tree-level scaling is not allowed for energies higher than the deviation of the Fermi level from van Hove singularity, let us write the phase space integration measure in terms of energy $(\xi)$ and polar angle $(\theta)$ variables

$$
\frac{1}{2 \pi} \int d \mathbf{k}=\frac{1}{2 \pi} \int d \xi d \theta J(\xi, \theta)
$$

with $J(\xi, \theta)=k(\xi, \theta) / v(\xi, \theta), k$ being the radial wave number and $v$ the group-velocity. Zeroth order (tree level) scaling tells us via power counting argument to consider $J(\xi, \theta)$ as function of $\theta$, neglecting any $\xi$ dependence about $\xi=0$, which is possible if $J(\xi, \theta)$ is an analytic function of $\xi$ at the whole shell $\pm \Lambda$ which, consequently, should contain no singularity.

We can also rescale the frequencies if $U\left(l_{c o}\right)$ is an analytic function of $\omega$ in the interval $\pm T_{c o}$ about the Fermi surface, which we assume to be the case 28 . In the scope of this treelevel scaling, as it has already been shown by Shankarथ, the slope of the electronic dispersion around the Fermi surface is irrelevant and the two marginal interactions correspond to two different constraints on the four-momenta in $U$. Since any $k_{\perp^{-}}$and $\omega$-dependence in $U$ is irrelevant, both marginal interactions depend only on coordinates of the zero frequency particles placed at the Fermi surface. For the first, "Fermi liquid" or forward interaction, the momenta satisfy the equation $\mathbf{k}_{1}=\mathbf{k}_{3}$, where the meaning of momenta can be seen from the equation (2.2). This interaction is slightly $\left(\sim U_{0}^{2}\right)$ renormalized by the high-energymodes $\left(l<l_{c o}\right)$, and is not involved in further renormalization. The second interaction is the pairing potential $V$, where the momenta satisfy the condition $\mathbf{k}_{1}=-\mathbf{k}_{2}$. The pairing $V$ depends only on angular coordinates of annihilated and created pairs. Keeping in mind the above remarks, we can write the action for the electrons in the ring $\pm T_{c o}$ around the Fermi surface as

$$
S=\int_{0}^{\infty} d \tau\left\{\sum_{\sigma} \int_{\epsilon<T_{c o}} \frac{d \epsilon}{2 \pi} \oint \frac{d s}{2 \pi v(\theta)} \bar{\Psi}_{\sigma}(\epsilon, \theta)\left(\partial_{\tau}-\epsilon\right) \Psi_{\sigma}(\epsilon, \theta)+\right.
$$




$$
\left.+\frac{1}{2} \sum_{\sigma \sigma^{\prime}} \int \frac{d \mathbf{q}_{e e}}{(2 \pi)^{2}} \oint \frac{d s}{2 \pi v(\theta)} \oint \frac{d s^{\prime}}{2 \pi v\left(\theta^{\prime}\right)} \overline{\hat{\Delta}}_{\sigma^{\prime}, \sigma, \mathbf{q}_{e e}}\left(\theta^{\prime}\right) V_{l=l_{c o}}\left(\theta, \theta^{\prime}\right) \hat{\Delta}_{\sigma^{\prime}, \sigma, \mathbf{q}_{e e}}(\theta)+F L\right\},
$$

where $\epsilon=k_{\perp} v(\theta)$, closed loop integrations are over the the Fermi surface and $F L$ stands for the effective Fermi liquid interaction. $\hat{\Delta}_{\sigma^{\prime}, \sigma, \mathbf{q}_{e e}}(\theta)$ is the energy-integrated number of pairs defined as

$$
\hat{\Delta}_{\sigma_{1}, \sigma_{2}, \mathbf{q}_{e e}}(\theta) \equiv \int_{\epsilon<T_{c o}} \frac{d \epsilon}{2 \pi} \Psi_{\sigma_{1}}(\mathbf{k}) \Psi_{\sigma_{2}}\left(-\mathbf{k}+\mathbf{q}_{e e}\right) \Theta\left(T_{c o}-\left|\left(-\mathbf{k}+\mathbf{q}_{e e}\right) \cdot \hat{\mathbf{n}} v(\theta)\right|\right) .
$$

Note that the integration measure over small momentum $\mathbf{q}_{e e}$ goes to zero as $l \rightarrow \infty$. A form

similar to (3.4) has been used by Weinberg25, but taking $V_{l=l_{c o}}\left(\theta, \theta^{\prime}\right)$ phenomenologically and not as the partially renormalized pairing interaction which we get from $U_{l=l_{c o}}\left(\theta_{1}, \theta_{2}, \theta_{3}\right)$ putting incoming particles 1 and 2 to $\theta$ and $\theta+\pi$ and outgoing 3 and 4 to $\theta^{\prime}$ and $\theta^{\prime}+\pi$. Note that the loop integration over $s$ can be understood as the scalar product over "vector components" of a "spin", where the number of components $N$ corresponds to $8 t / T_{c} 2922$. The integrations have the weight factor $1 / v(\theta), v(\theta)$ being the anisotropic Fermi velocity, what suggests to introduce a new angular coordinate

$$
z(\theta)=\frac{\int s \frac{d s}{v(\theta)}}{2 \pi N_{F}}
$$

where $N_{F}$ is the density of states at the Fermi level. The function $z(\theta)$ is shown in Fig.4 for few different values of $\mu$. Starting from the new Hamiltonian (3.4) we can calculate now the function $\beta_{e e}\{V\}$ in $z$-space and obtain the flow-equation

$$
\partial_{l} V\left(z, z^{\prime}\right)=-\frac{N_{F}}{2 \pi} \oint d z^{\prime \prime} V\left(z, z^{\prime \prime}\right) V\left(z^{\prime \prime}, z^{\prime}\right)
$$

where the coordinate $z$ appears instead of $\theta(z)$. For initial condition we take $V_{l=l_{c o}}\left(\theta(z), \theta\left(z^{\prime}\right)\right)$.

\section{DIAGONALIZATION OF THE PAIRING POTENTIAL}

To make the differential equation (3.7) solvable one has to diagonalize the pairing potential $V\left(z, z^{\prime}\right)$ 24 25 Since it is invariant under all symmetry elements of the $D_{4}$ point group, 
its most general form in $z$-space can be written as

$$
V\left(z, z^{\prime}\right)=\sum_{\gamma} \sum_{m, n} V_{m, n}^{\gamma} f_{m, n}^{\gamma}\left(z, z^{\prime}\right)
$$

where $V_{m n}^{\gamma} \equiv\langle m \gamma|V| n \gamma\rangle$ and $f_{m, n}^{\gamma}\left(z, z^{\prime}\right) \equiv\langle m \gamma \mid z\rangle\left\langle n \gamma \mid z^{\prime}\right\rangle$. The function $\langle m \gamma \mid z\rangle$ is the $m^{\text {th }}$ basis state of the $\gamma$-representation of the point group $D_{4}$. It is proportional to the function $\cos 4 m z, \sin 4 m z, \cos (4 m+2) z, \sin (4 m+2) z$, and $[\cos (2 m+1) z \pm \sin (2 m+1) z]$, for $\gamma=A_{1}, A_{2}, B_{1}, B_{2}$, and $E$ respectively. Using (4.1), the flow equation (3.7) becomes

$$
\partial_{l} V_{m, n}^{\gamma}=-\frac{N_{F}}{2 \pi} \sum_{\nu} V_{m, \nu}^{\gamma} V_{\nu, n}^{\gamma}
$$

with the initial condition

$$
V_{m, n}^{\gamma}\left(l=l_{c o}\right)=\int d z d z^{\prime} f_{m, n}^{\gamma}\left(z, z^{\prime}\right) V_{l_{c o}}\left(z, z^{\prime}\right)
$$

To solve exactly the equation (4.2) one has to diagonalize five infinite dimensional matrices $V_{m, n}^{\gamma}\left(l=l_{c o}\right)$, thus decoupling completely the flow (4.2) into a set of differential equations whose solution is

$$
V_{\lambda}^{\gamma}(l)=\frac{V_{\lambda}^{\gamma}\left(l_{c o}\right)}{1+\left(\frac{N_{F} V_{\lambda}^{\gamma}\left(l_{c o}\right)}{2 \pi}\right)\left(l-l_{c o}\right)} .
$$

Here $\lambda$ labels the eigenvalues within the representation $\gamma$. If $V_{\lambda}^{\gamma}\left(l_{c o}\right)$ is negative, the denominator has a zero at $l=l_{c}(\gamma, \lambda)$ and an instability occurs.

The renormalization group calculation of the pairing interaction $V\left(l_{c o}\right)$ is an extremely difficult problem because of the interplay between the cutoff $\Lambda$ and the geometry of the two-dimensional shells $d \Lambda$. However for some range of $\langle n\rangle$ and $U_{0}$ (essentially small $U_{0}$ and $\langle n\rangle$ not too close to half-filling) the problem can be reduced to only one simple integration, i.e.

$$
V\left(l_{c o}\right) \approx U_{0}+I_{0}
$$

with

$$
I_{0}=U_{0}^{2}\left(P_{e e}\left(l_{c o}\right)+P_{e h}\left(l_{c o}\right)\right)
$$


where $P_{e e}(l)$ is the partially integrated e-e loop, defined in a same way as $P_{e h}(l)$ in eq.(3.1). $P_{e h}(l)$ comes just from the last diagram in the expression for $\Gamma_{4}$ in Fig. 1, because the other three e-h loops cancel exactly for any $U$ symmetric under exchange. The approximation (14.5) is allowed if $I_{0} / U_{0}$ is a small parameter. A good estimate of the magnitude of this parameter is given by the $A_{1}$-part of $U_{0} P_{e h}\left(l_{c o}\right)$, giving the criterion for the validity of our approach in $\left(\langle n\rangle, U_{0}\right)$-space. The line $I_{0}(\mu) / U_{0} \sim 1$ is shown in Fig. 5 . Below the line the approximation (4.6) is justified.

By definition of $l_{c o}, P_{e h}\left(l_{c o}\right) \approx P_{e h}(l=\infty)$. Moreover, $P_{e e}\left(l_{c o}\right)$ has no dependence on $z$ and $z^{\prime}$, since it depends on external momenta only through $\mathbf{k}_{\mathbf{1}}+\mathbf{k}_{\mathbf{2}}$, which we put to zero. Consequently, its only nonzero component is $\left\langle 0 A_{1}\left|P_{e e}\right| 0 A_{1}\right\rangle$. Thus, for the calculation of all other components of $V_{l_{c o}}$ we use just the bubble $P_{e h}(l \rightarrow \infty)$ with the momentum transfer $\mathbf{q}=\mathbf{k}_{F}(z)-\mathbf{k}_{F}\left(z^{\prime}+\pi\right)$. Fig.6(a) shows $P_{e h}(l \rightarrow \infty)$ as a function of $z$ and $z^{\prime}$ for chemical potential $\mu / 4 t=-0.2$.

The minimal eigenvalues of $V\left(l_{c o}\right)$ in all five channels, named $\lambda_{\min }^{\gamma}$, are shown in Fig.6(b) as functions of $\mu$. These curves indicate which kind of superconducting symmetry becomes critical at some given $\mu$. The eigenvalues for each channel are calculated taking only the first four harmonics for $A_{1}, A_{2}, B_{1}$ and $B_{2}$, and the first six harmonics for the $E$ representation. The corresponding eigenvectors determine the Fourier spectrum of the gap function. A very important result is that the relevant harmonic of the superconducting fluctuations in the $B_{1}$ channel occurs very close to just $\cos (2 z)$, being thus determined only by the structure of the Fermi surface and not by the interaction. Fig. 7(a) show the instable order parameters $\Delta_{B_{2}}$ for $\mu=-0.5, \Delta_{E}$ for $\mu=-0.31$ and $\Delta_{B_{1}}$ for $\mu=-0.001$ as a function of the Fermi surface angle $\theta$. The evolution of the function $\cos 2 z(\theta)$ (i.e. the first harmonic of $B_{1}$ ) with $\log (-\mu)$, given only by the dependence of $z$ on $\theta$, is shown in Fig. 7(b). The strength of the peaks near the van Hove points increases and the magnitude in the area between the peaks decreases with $\log (-\mu)$. 
The critical temperature is given by a cutoff for which the most attractive diagonal component of $V$ diverges, i.e.

$$
T_{c}=8 t \exp \left[-l_{c}\left(\gamma, \lambda_{\min }\right)\right]
$$

where $\lambda_{\min }=\min \left\{\lambda_{\min }^{\gamma}\right\}$. Fig.8. shows $l_{c}$ as a function of $\log (\mu)$. The critical temperature decreases extremely fast as we go away from the half-filling. An increase of $U_{0}$ could save the situation, but in that case our perturbative method ceases to be sufficient (see Fig. 5). Since the cuprates are superconductors for fillings quite far from one electron per site $(\langle n\rangle \sim 1-0.17)$, this result means that the small- $U$ Hubbard model cannot describe these systems quantitatively. However, the model gives very precious informations about the form of the gap function in the $B_{1}$-instable regime, which will not change considerably with increasing $U_{0}$, as long as $\cos (2 z(\theta))$ is the dominant attractive harmonic in $V\left(l_{c o}\right)$.

\section{MIXED-SYMMETRY SUPERCONDUCTIVITY}

Once the renormalization flow has been integrated for $l<l_{c o}$, assuming that the interaction did not diverge earlier in the antiferromagnetic channel, the detailed angular dependence of the superconducting gap function can be easily found. In general, a superconducting state with the symmetry corresponding to the lowest of the eigenvalues $\lambda_{\min }^{\gamma}$ will be formed. However, when two of the $\lambda_{\min }^{\gamma}$ are close to each other, a more complicated situation can occur: for definiteness, consider the region $0.206<|\mu / 4 t|<0.276$ in Fig.6(b), where the $B_{2}$ eigenvalue is the most attractive after the $B_{1}$. Let us suppose that $B_{1}$ order of the simplest form $\Delta_{B_{1}} \sim \cos 2 z$ has formed and that the temperature is close to $T_{c}$. Among the remaining symmetry channels, $B_{2}$ is the only one which can give a large gap function in the node-points of $\Delta_{B_{1}}$ and zero in the points where $\Delta_{B_{1}}$ is maximal. Consequently, we expect that the flow of the type (4.4) with $\gamma=B_{2}$ will not be strongly affected by the existing $B_{1}$ order. Considering to a first approximation the two flow equations $\left(\gamma=B_{1}\right.$ and $\left.\gamma=B_{2}\right)$ as independent, and taking only the first harmonics of the $B_{1}$ and $B_{2}$ representations, we can construct the 
relevant part of the pairing interaction which gives two phase transitions, one with $B_{1}$ and the other with $B_{2}$-symmetry:

$$
V_{l_{c o}}\left(\theta_{1}, \theta_{2}\right)=V^{\left(B_{1}\right)} \frac{1}{\pi} \cos 2 z\left(\theta_{1}\right) \cos 2 z\left(\theta_{2}\right)+V^{\left(B_{2}\right)} \frac{1}{\pi} \sin 2 z\left(\theta_{1}\right) \sin 2 z\left(\theta_{2}\right),
$$

where all details of the Fermi surface are contained in the dependence of $z$ on $\theta$. From eq. (4.4) one finds that the ratio between two critical temperatures is given by $T_{c}^{\prime} / T_{c}=$ $\exp \left[-2 \pi\left(1 / V^{\left(B_{1}\right)}-1 / V^{\left(B_{2}\right)}\right) / N_{F}\right]$. From Fig.6(b) note that the ratio $T_{c}^{\prime} / T_{c}$ is very sensitive to the variation of the chemical potential. The gap function resulting from (5.1) has the form

$$
\Delta(\theta)=\Delta_{B_{1}} e^{i \phi_{1}} \cos 2 z(\theta)+\Delta_{B_{2}} e^{i \phi_{2}} \sin 2 z(\theta)
$$

where $\Delta_{B_{1}}, \Delta_{B_{2}}, \phi_{1}$ and $\phi_{2}$ are real. These parameters can be determined minimizing the mean-field expression for the free energy per site 30

$$
F=-\frac{2 T}{N} \sum_{\mathbf{k}} \Theta\left(T_{c o}-\left|\xi_{\mathbf{k}}\right|\right) \ln \cosh \frac{E_{\mathbf{k}}}{2 T}+\left|\Delta_{1}\right|^{2} / V^{\left(B_{1}\right)}+\left|\Delta_{2}\right|^{2} / V^{\left(B_{2}\right)}
$$

where $E_{\mathbf{k}} \equiv \sqrt{\xi_{\mathbf{k}}^{2}+|\Delta(\theta)|^{2}}$ and the theta function constrains the momentum summation to run only over the states within the energy shell $\pm T_{c o}$ about the Fermi surface. The minimization of $F$ with respect to $\cos \left(\phi_{1}-\phi_{2}\right)$ gives

$$
\phi=\phi_{1}-\phi_{2}= \pm \frac{\pi}{2}
$$

i.e. the resulting gap function is of the type $B_{1} \pm i B_{2}$. It is interesting to remark that the same kind of gap function has been obtained by Laughlin using the anyon picture 31 The particularity of this gap function (and of any gap consisting of two different symmetry terms with a phase difference of $\pm \pi / 2$ ) is that it breaks time reversal symmetry.

We can now try to understand recent ARPES measurements by Jian Ma and coworkers 9 on the Bi2212 compound. From their experiment it appears that two superconducting instabilities occur; the first one is at $T=T_{c}$ and has probably the $B_{1}$ symmetry. The second instability occurs at $T_{c}^{\prime}=0.81 T_{c}$; it introduces a nonzero gap at the points $\theta=(2 n+1) \pi / 4$, i.e. halfway between the corners of the half-filled Fermi. The function measured by ARPES 
is $|\Delta(\theta)|$ and in the picture discussed above has no zeros and minima on the diagonals of the Brillouin zone if both $\Delta_{B_{1}}$ and $\Delta_{B_{2}}$ are finite, $\left|\Delta_{B_{1}}\right|>\left|\Delta_{B_{2}}\right|$ and $\phi= \pm \pi / 2$. This is in agreement with the experiments because the gap in the diagonal direction is just equal to $\Delta_{B_{2}}$, introduced at $T=T_{c}^{\prime}$. The minimum of $|\Delta(\theta)|$ on the diagonals is in agreement with other ARPES experiments 6 . closeness of two different $\lambda$ 's only occurs in very narrow parts of the parameter space and therefore to a certain degree is accidental.

\section{CONCLUSIONS}

We have formulated the one-loop renormalization group for a two-dimensional system of interacting electrons on a square lattice, described by the Hubbard model. For band filling different from one-half, the renormalization flow for $l$ superior to some crossover $l_{c o}$ comes only from the contribution due to the electron-electron bubble-diagram, while the electronhole contribution decays exponentially as $\exp (-l / 2)$, where $l_{c o}$ depends on band filling, but is independent on the strength of the interaction. We decompose the BCS pairing interaction $V$ for electrons in the vicinity of the Fermi surface in Fourier components of the five irreducible representations of the $D_{4}$ point group, defined at the Fermi surface. Diagonalizing $V\left(l_{c o}\right)$ in each representation, we get five sets of decoupled BCS flow equations. The minimal (i.e. the most negative) eigenvalue of $V\left(l_{c o}\right)$ determines the critical temperature and the eigenvector gives the form of the gap function. Unlike the usual approach, 2 the characteristic of the procedure presented here to obtain the symmetry of the gap function is that only the axial coordinates at the Fermi surface is relevant, while the radial dependence is "scaled out". Moreover, the renormalization group treatment of the whole Brillouin zone, and not only

of the narrow belt about the Fermi surface has allowed us to show that the origin of the attractive part of the pairing interaction in the Hubbard model is in electron-hole fluctuations on rather high energy scales, up to the bandwidth.

We have calculated $V\left(l_{c o}\right)$ for the case where the flow due to the electron-hole channel 
can be treated perturbatively, i.e. when the filling is far enough from one-half. The diagonalization of $V$ in terms of angular harmonics gave us the type of superconducting instability: for weak filling, the instability occurs in the $B_{2}\left(d_{x y}\right)$ singlet channel, while for filling close to one-half, the $B_{1}\left(d_{x^{2}-y^{2}}\right)$ singlet instability strongly overwhelms all others, what are the results in agreement with previous work.11,16.17 Particularly interesting is the fact that the order parameter $\Delta_{B_{1}}(\theta)$ can be very well approximated by the function $\cos 2 z(\theta)$, where the function $z(\theta)$ depends only on the anisotropic Fermi velocity and on the geometry of the Fermi surface. This gives for $\Delta_{B_{1}}(\theta)$ a function that has peaks in the directions of the van Hove singularities. The slope of the peaks increases as we approach half-filling. This can be a justification to consider the interaction only between electrons in the close vicinity of the van Hove points as relevant if we are very close to half-filling 20 . We believe that the form of $\Delta_{B_{1}}(\theta)$ does not depend considerably on the strength of interaction, and recent T-matrix calculations 33 for realistic values of the interaction $U_{0}$ give a gap function in accord with our assumptions.

We find a superconducting instability at any electron concentration away from halffilling. The underlying physical mechanism, namely exchange of spin or charge density fluctuations, is the same as in previous approaches.13-19 We do however feel that our present results are on a more solid footing than the previous work because the present one-loop renormalization group scheme does not make any a priori assumptions about important or unimportant diagrams and provides a more systematic way of handling the dynamics of the fluctuations being exchanged. The only restrictions come from (i) the limitation to oneloop order, necessitating weak coupling, and (ii) the requirement that the e-h diagram are a perturbation with respect to the e-e diagrams, implying that we can not be too close to half-filling. The region of validity of the approach is shown in Fig.5. Further, self-energy diagrams have been neglected, however, these are expected to produce important effects only at two-loop order, and therefore are expected to be negligible in weak coupling.

In our weak coupling model the superconducting critical temperature is negligible (but it exists, for any filling!) if we are not in the immediate vicinity of half-filling, which means that 
the Hubbard model with small $U_{0}$ and small (perturbative) antiferromagnetic fluctuations does not suffice to describe the high critical temperature $(\sim 0.02 t)$ of the cuprates. There exist two possible ways (related to two restrictions of our calculations) to increase the critical temperature. The first is to simply increase $U_{0}$ and to remain far from the half-filling, keeping the e-h channel non-singular. To treat this case, an approach perturbative in $U_{0}$ is possibly only of limited use. Ideally, renormalization should be done exactly, and not using a simple one-loop (or $n$-loop) scheme (which is actually just an "intelligent" version of the perturbative summation). We can speculate and suppose that even in the case of strong coupling there exists the crossover $\tilde{l}_{c o}$, above which the flow is of the BCS type. It is to expect that $\tilde{l}_{c o}$ is not very different from $l_{c o}$ that we have calculated. This means that the cutoff $\tilde{T}_{c o}$ for the effective BCS theory is $\Lambda_{0} \exp \left(-l_{c o}\right)$ (see Fig.3. and eq.(3.2)), where $\Lambda_{0}$ is the initial cutoff of the theory, equal to $8 t$. The second possibility to increase $T_{c}$ is to approach half-filling very closely, making Fermi surface nesting important but remaining in a weakcoupling regime. For that case, a simplified one-loop renormalization group calculation $\$ 20$ has shown that superconductivity wins over antiferromagnetism only if the e-h contribution to the flow decays before the divergence in the antiferromagnetic channel takes place. Thus, we can say that here too, the effective theory is of BCS type. The difference with the first scenario is that the effective cutoff is very small (Fig. 3), and that the coupling constant is very strong, due to the strong flow in both e-e and e-h channels at all scales $l<l_{c o}$. A very important feature of the nested case with a small $U_{0}$ is that it can be treated in terms of the one-loop renormalization group, renormalizing $U$ as a function only of three angular variables. This is allowed because all important physics (i.e. the majority of the e-e and e-h flow) is contained in the vicinity of the Fermi surface, making the effective phase space to be a rather narrow square ring $\pm \Lambda_{i} ;\left(8 t \gg \Lambda_{i} \gg \Lambda_{c o}\right)$ where the marginally relevant interaction is a function only of the angular position of the particles on the ring.

Finally, we have discussed the possibility of a superconducting state with a mixed symmetry. In the presence of $B_{1}$ order, the flow in the $B_{2}$ channel (which is the second most attractive one for $0.206<\mu<0.276$ ) will be only weakly affected by a nonzero order param- 
eter of $B_{1}$ symmetry. This gives rise to two superconducting instabilities, with the critical temperatures $T_{c}$ for the $B_{1}$ and $T_{c}^{\prime}$ for the $B_{2}$ channel, and $T_{c}^{\prime}<T_{c}$. We have given the form of the pairing function for the effective BCS theory. At $T<T_{c}^{\prime}$ the relative phase of two order parameters is $\phi= \pm \pi / 2$. The resulting form of the energy gap $|\Delta(\theta)|$ has no zeros and minima are in diagonal directions, providing a possible qualitative explanation of ARPES experiments by Shen 7 and the decrease of the anisotropy with decreasing $T .9$

\section{ACKNOWLEDGMENTS}

We are indebted to N. Dupuis and L. Guerrin for a number of helpful discussions. This work was supported by EC contract no. ERBCHRXCT 940438. 


\section{REFERENCES}

${ }^{1}$ For recent short overview see: J. R. Schrieffer, Solid State Commun. 92, 129 (1994).

${ }^{2}$ D. A. Wollman et al., Phys. Rev. Lett. 74, 797 (1995).

${ }^{3}$ J. R. Kirtley et al., Nature 373, 225 (1995).

${ }^{4}$ R. C. Dynes, Solid State Commun., 92, 53 (1994).

${ }^{5}$ W. N. Hardy et al., Phys. Rev. Lett. 70, 3999 (1993).

${ }^{6}$ J. A. Martindale et al., Phys. Rev. B47, 9155 (1993).

${ }^{7}$ Z.-X. Shen et al., Science 267, 343 (1995).

${ }^{8}$ M. R. Norman, M. Randeria, H. Ding, J. C. Campuzano, and A. F. Bellman, preprint (submitted to SISSA, cond-mat 9507021).

${ }^{9}$ Jian Ma et al., Science 267, 862, (1995).

${ }^{10}$ W. Kohn and J. M. Luttinger, Phys. Rev. Lett. 15, 524 (1965).

${ }^{11}$ M. A. Baranov, A. V. Chubukov, and M. Yu. Kagan, Int. J. Mod. Phys B6, 2471 (1992).

12 N. Bulut, D. J. Scalapino, and S. R. White, Phys. Rev. B50, 9623 (1994).

${ }^{13}$ V. J. Emery, Synt. Met. 13, 21 (1986).

${ }^{14}$ K. Miyake, S. Schmitt-Rink, and C. M. Varma, Phys. Rev. B34, 6554 (1986).

${ }^{15}$ D. J. Scalapino, E. Loh, Jr. and J. E. Hirsch, Phys. Rev. B34, 8190 (1986).

${ }^{16}$ N. E. Bickers, D. J. Scalapino and R. T. Scalettar, Int. J. Mod. Phys. B1, 687 (1987).

${ }^{17}$ N. E. Bickers, D. J. Scalapino and S. R. White, Phys. Rev. Lett. 62, 961 (1989).

${ }^{18}$ N. E. Bickers and D. J. Scalapino, Ann. Phys. (N.Y.) 193, 206 (1989).

${ }^{19}$ N. E. Bickers and S. R. White, Phys. Rev. B43, 8044 (1991). 
${ }^{20}$ H. J. Schulz, Europhys. Lett. 4, 609 (1987).

${ }^{21}$ I. E. Dzyaloshinskii, Sov. Phys. JETP66, 848 (1988).

${ }^{22}$ I. E. Dzyaloshinskii and V. M. Yakovenko, Sov. Phys. JETP67, 844 (1988).

${ }^{23}$ A. Georges and J. S. Yedidia, Phys. Rev. B43, 3475 (1991).

${ }^{24}$ R. Shankar, Rev. Mod. Phys. 66, 129 (1994).

25 S. Weinberg, Nucl. Phys. B413, 567 (1994).

${ }^{26}$ H. J. Schulz, in "Low-Dimensional Conductors and Superconductors", eds. D. Jérome and L. G. Caron, (Plenum, New York, 1987).

${ }^{27}$ J. W. Negele and H. Orland, "Quantum Many-Particle Physics", Addison Wesley (1988).

28 The four point vertex is not an analytic function of momenta and frequency, because the e-h bubble has a singularity at $\omega=q=0$. This aspect is of importance for the Fermi liquid properties (for details see: N. Dupuis and G. Y. Chitov, preprint), and since we are interested in logarithmic instabilities of the model, we can ignore it.

${ }^{29}$ S. K. Ma, Modern Theory of Critical Phenomena, Benjamin (1976).

${ }^{30}$ G. Kotliar, Phys. Rev. B37, 3664 (1987).

${ }^{31}$ R. B. Laughlin, Physica C234, 280 (1984).

${ }^{32}$ G. E. Volovik and L. P. Gor'kov, Sov. Phys. JETP 61, 843 (1985).

33 T. Dahm and L. Tewordt, Phys. Rev. Lett. 74, 793 (1995). 


\section{FIGURES}

FIG. 1. The one-particle irreducible diagrams for the vertices $\Gamma_{2}$ and $\Gamma_{4}$, generating the renormalization of the self-energy and the interaction, respectively.

FIG. 2. The e-h bubble (solid line) and its derivative over $l$ (dashed line) for $\mu / 4 t=-0.25$ (a) and $\mu / 4 t=-0.02(\mathrm{~b})$, for momentum transfer $\mathbf{q}_{e h}=2 \mathbf{k}_{F} \|(\pi, \pi)$.

FIG. 3. The scale $l_{x}$ as a function of filling $\langle n\rangle$. For $l>l_{x}$ the e-h flow decays exponentially. The insert shows the relation between $\mu$ and $\langle n\rangle$.

FIG. 4. The relation between the angular variable $z$ and the observable polar angle $\theta$ for $-\mu / 4 t=2 \times 10^{-n} ; n=1(a), 2, \ldots, 9(i)$.

FIG. 5. The curve $U_{0} P_{e h}(l \rightarrow \infty)=1$. Below the curve, the e-h contribution to the renormalization can be treated perturbatively.

FIG. 6. (a) The shape of the function $P_{e h}(l \rightarrow \infty)$ in $\left(z, z^{\prime}\right)$ space at $-\mu / 4 t=0.2$. The nesting at half filling occurs for $z=z^{\prime}=\pi / 4$. The split singular lines show the best incommensurate nesting vector. (b) The minimal eigenvalue of the pairing $V\left(z, z^{\prime}\right)$ in every of 5 irreducible representations of $D_{4}$ point group.

FIG. 7. (a) The shape of three possible gap functions: $\Delta_{B_{1}}(\theta)$ for $\mu / 4 t=-0.001$ (dotdashed); $\Delta_{B_{2}}(\theta)$ for $\mu / 4 t=-0.5$ (dashed) and $\Delta_{E}(\theta)$ for $\mu / 4 t=-0.31$ (solid line). (b) A very good approximation for $\Delta_{B_{1}}(\theta)$ is just $\cos 2 z(\theta)$, shown here for the same choice of $\mu$ as in fig. 4. The dashed line shows $\cos \theta$ to comparison.

FIG. 8. The scale $l_{c}=-\ln T_{c} / 8 t$ as a function of the logarithm of the chemical potential, for a few values of coupling $U_{0}$. For very small $\mu$ and for $U_{0} / 4 t>0.5$ the curves are out of the range of validity (see fig.5). 

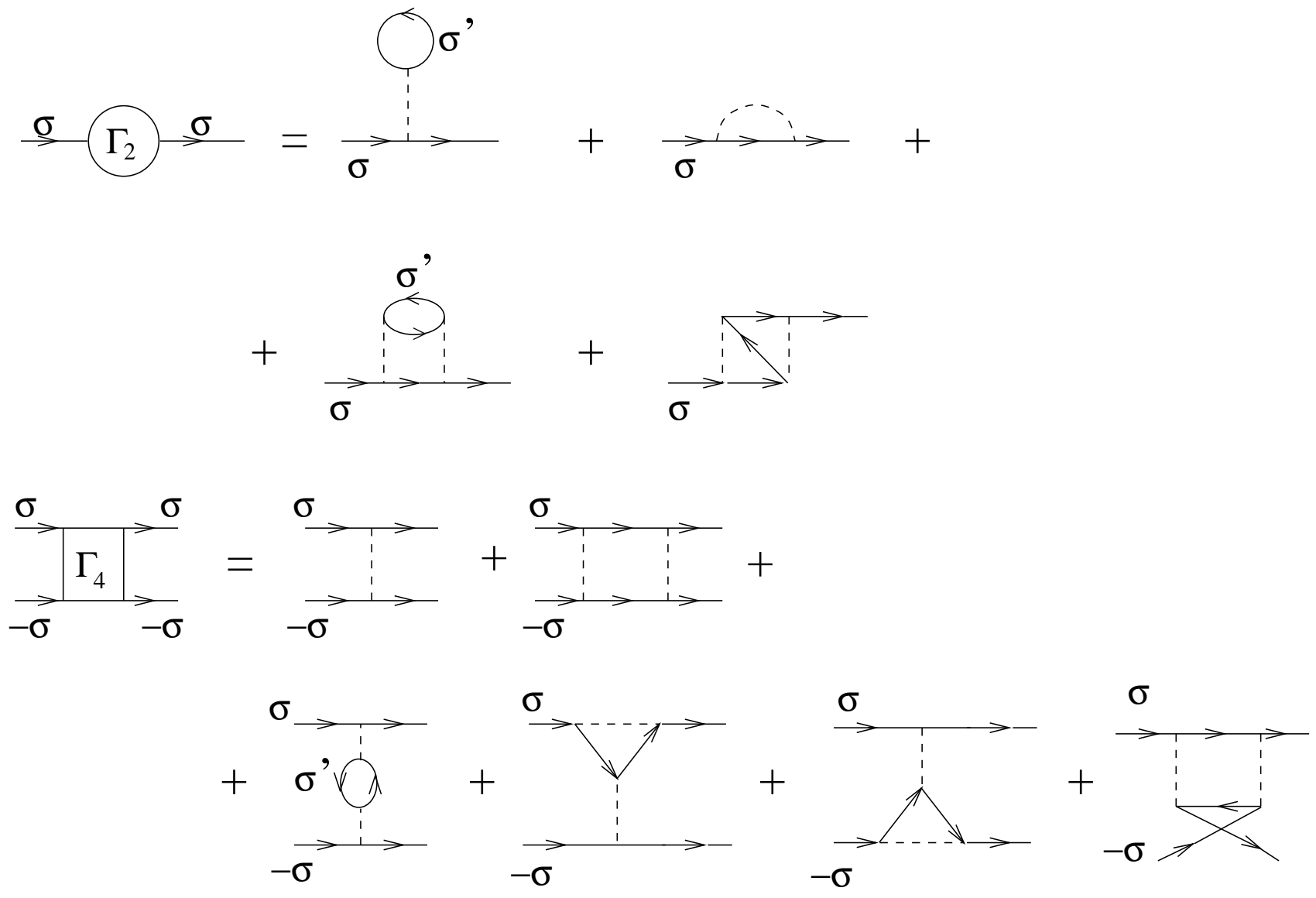
(a)

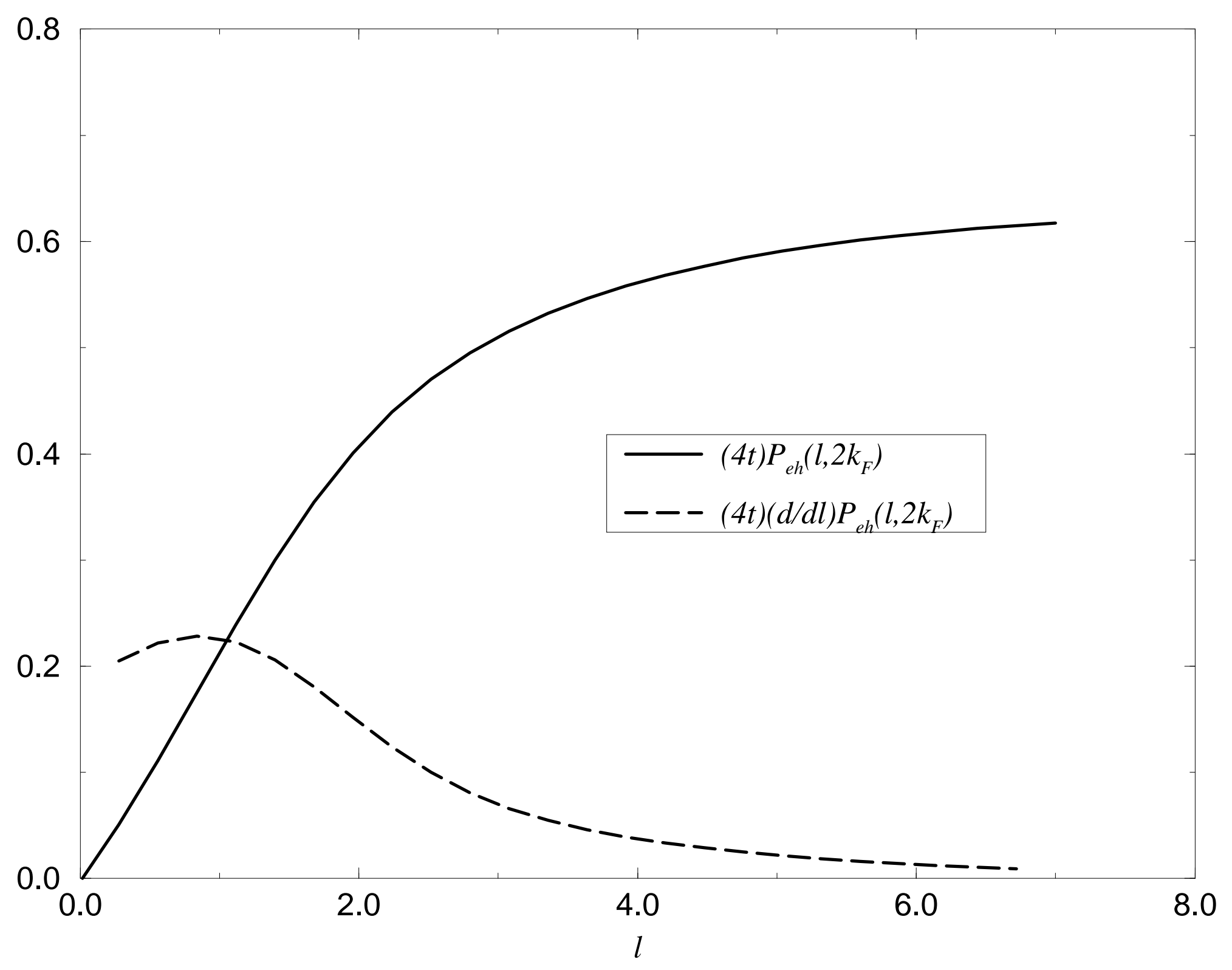


(b)

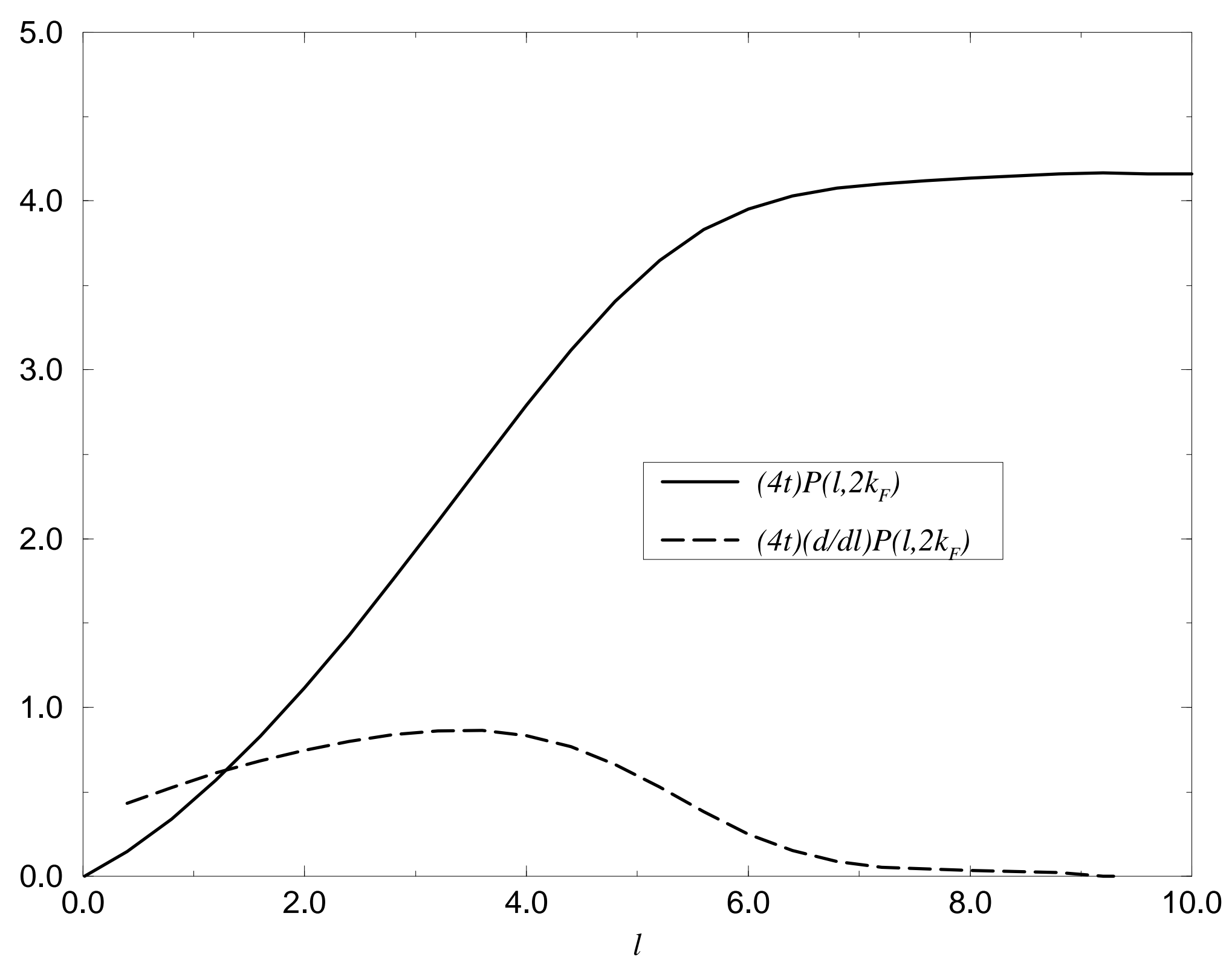




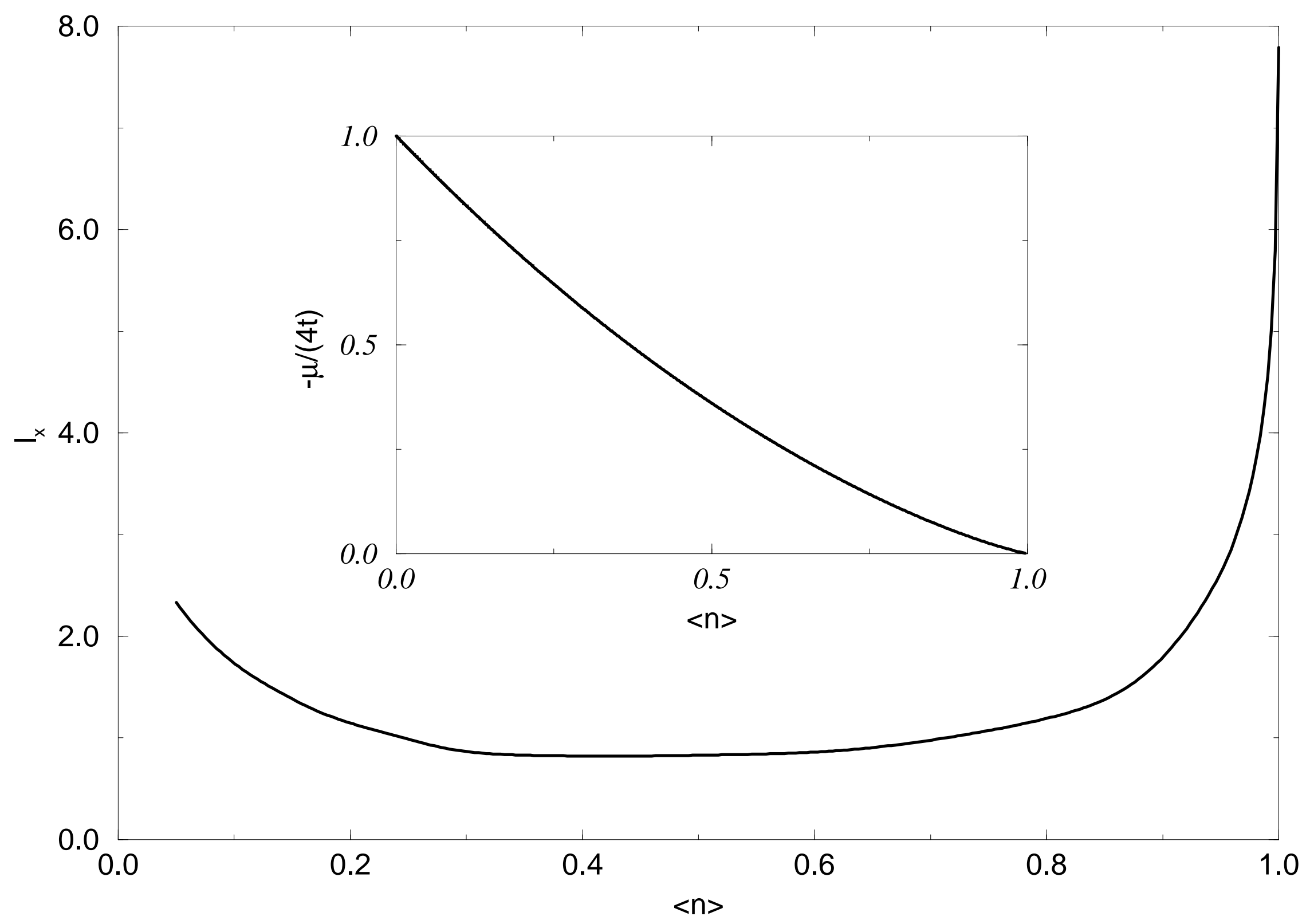




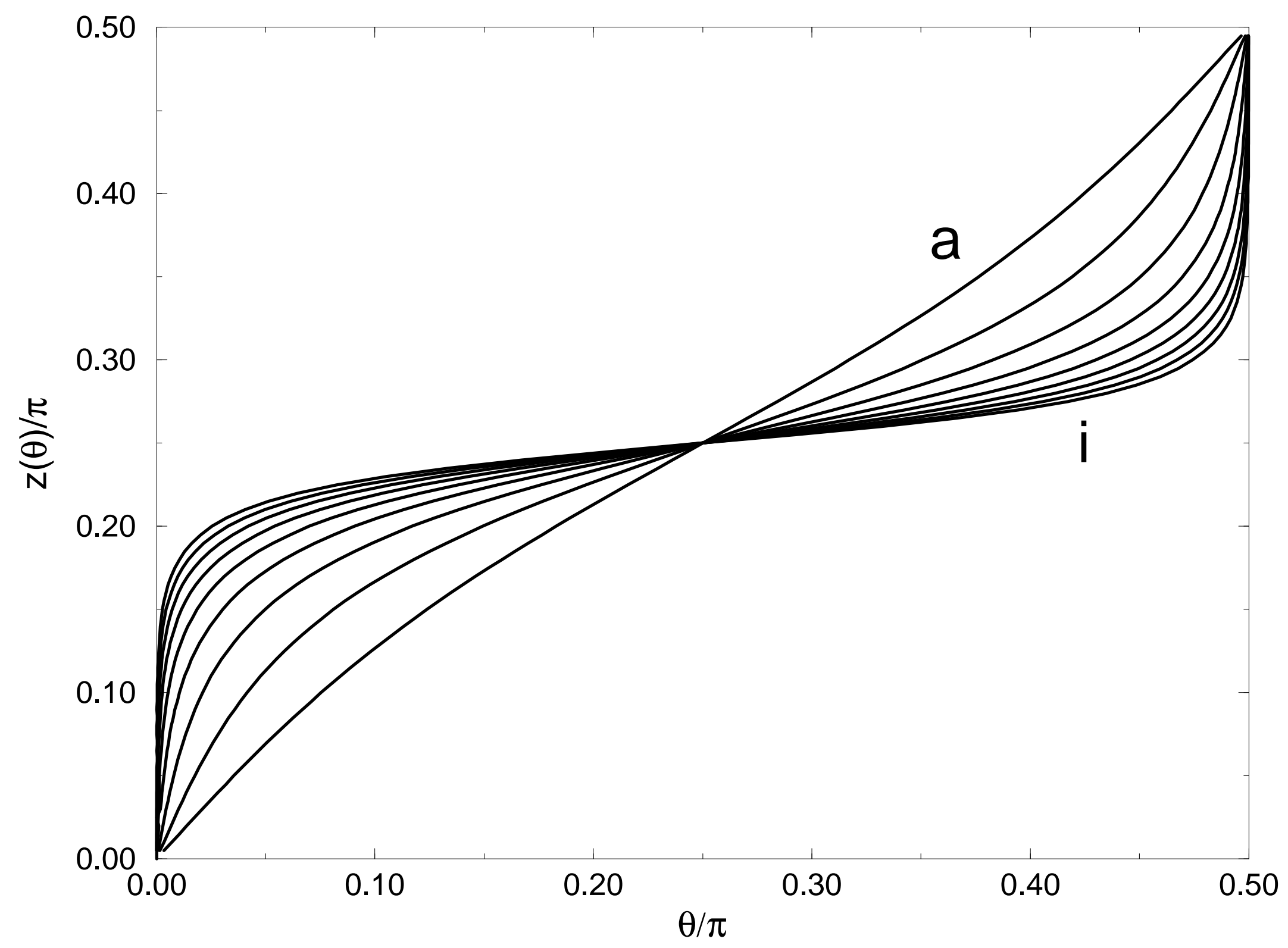




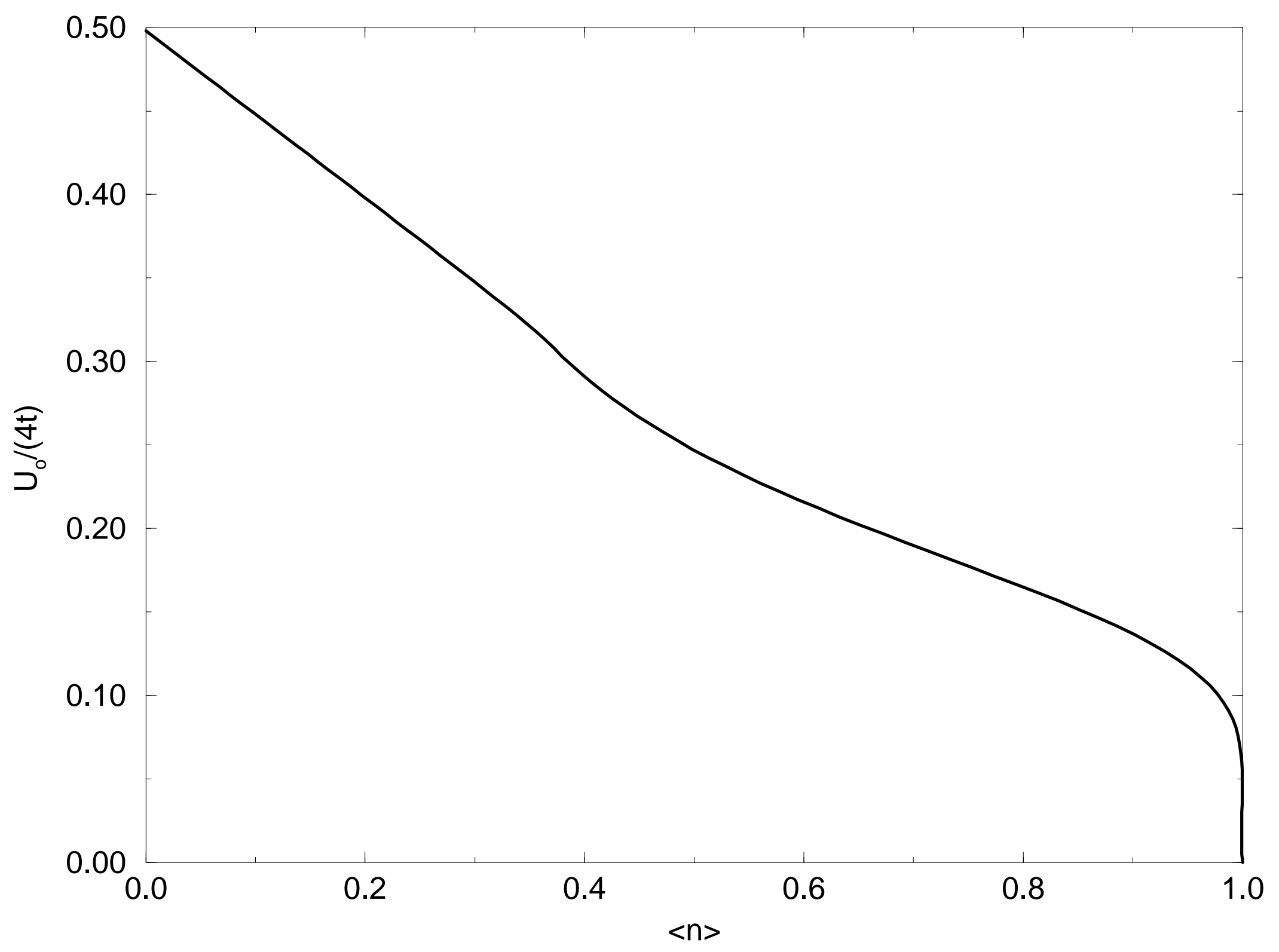




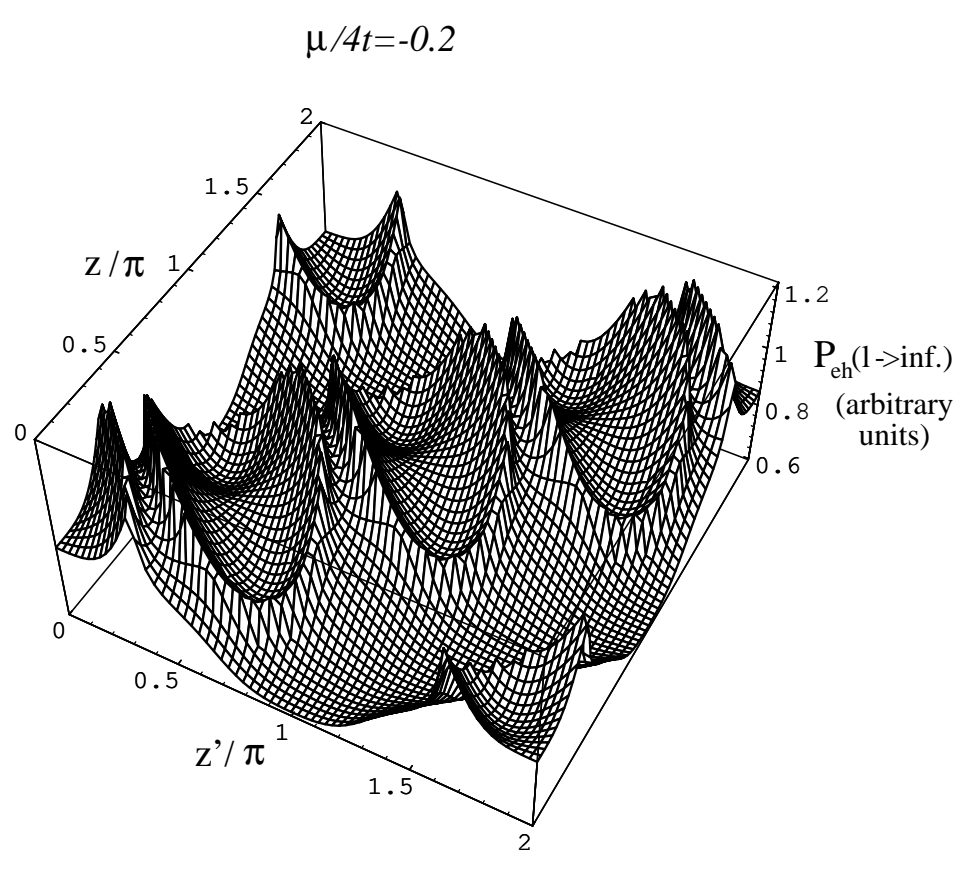


(b)

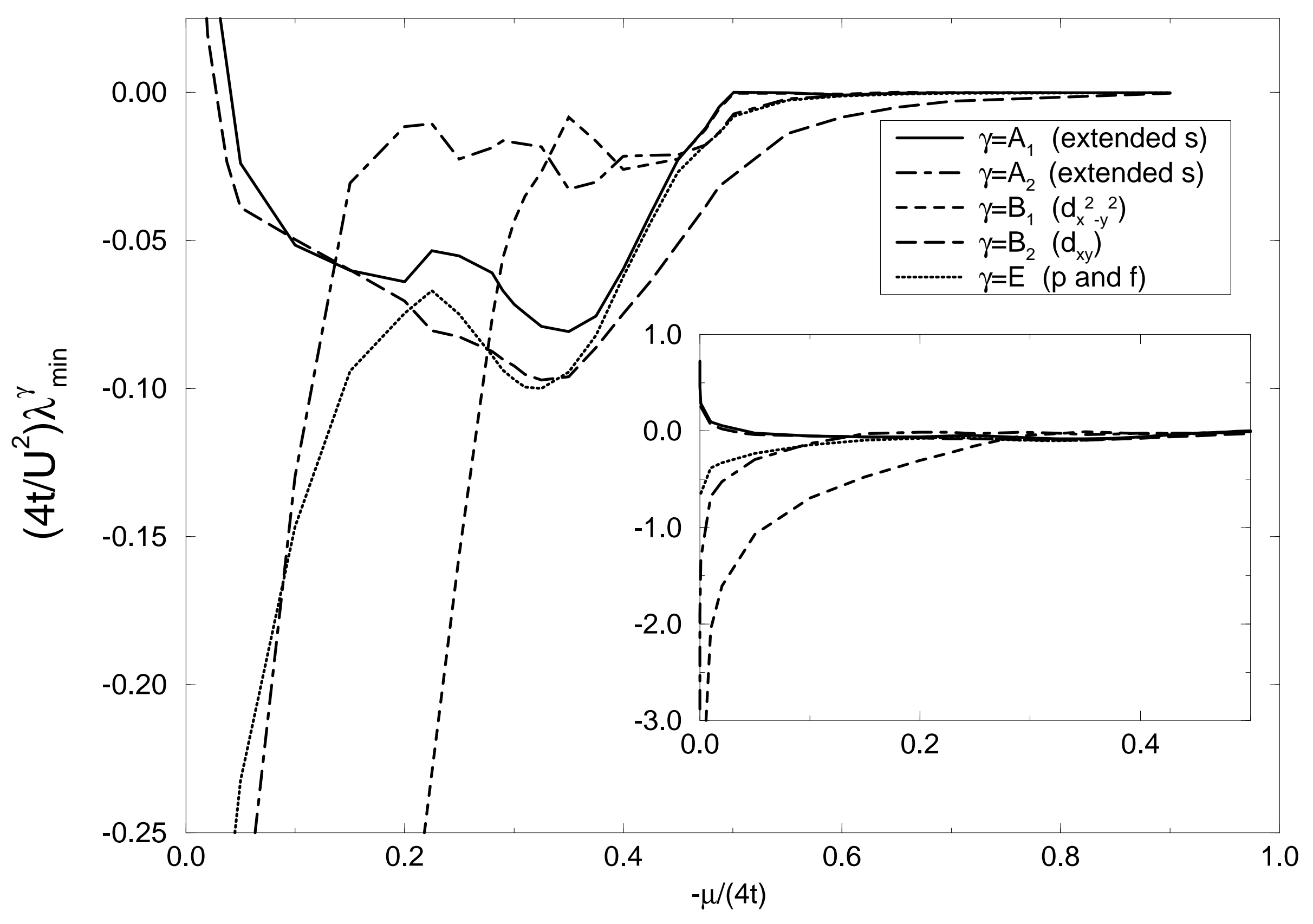


(a)

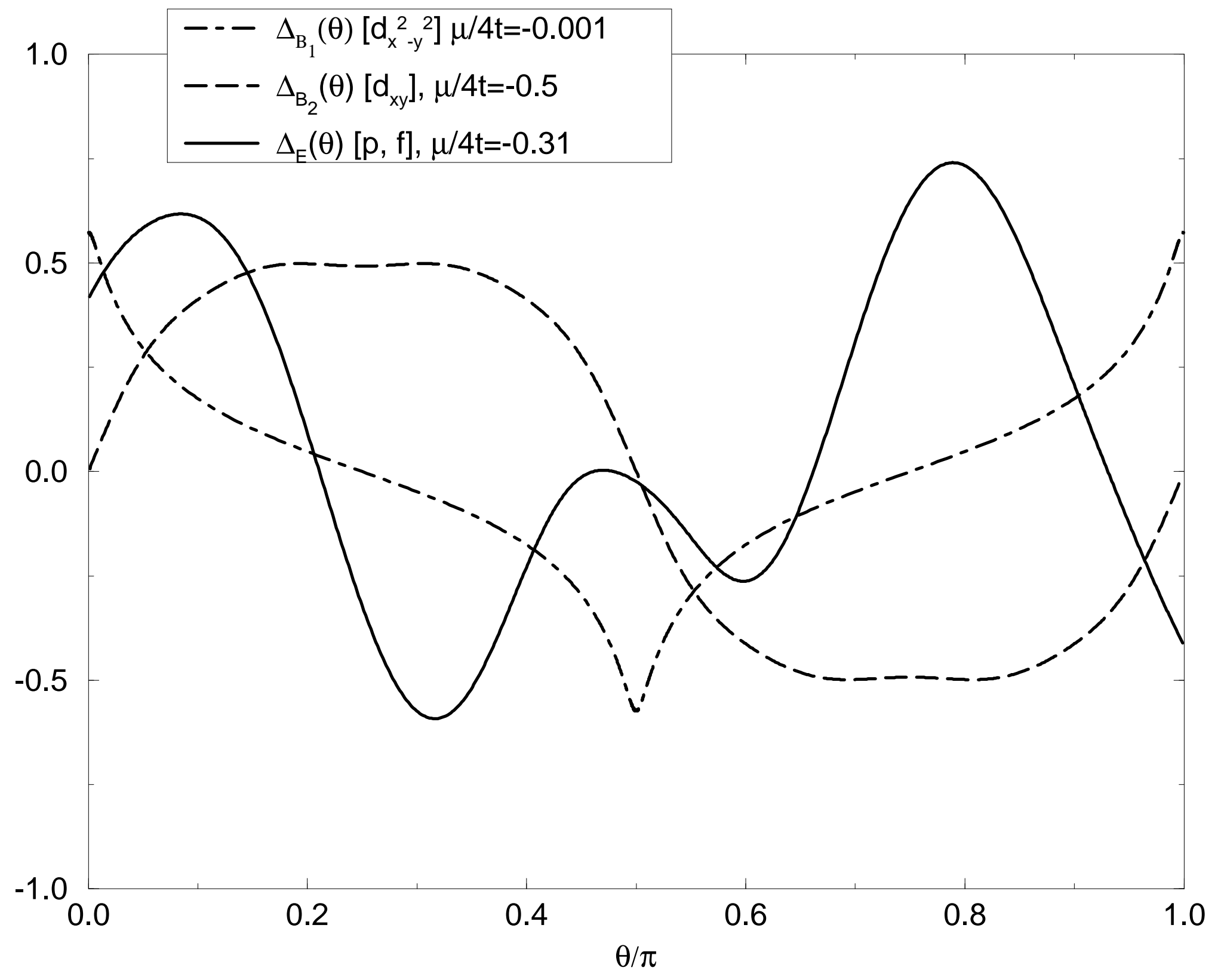


(b)

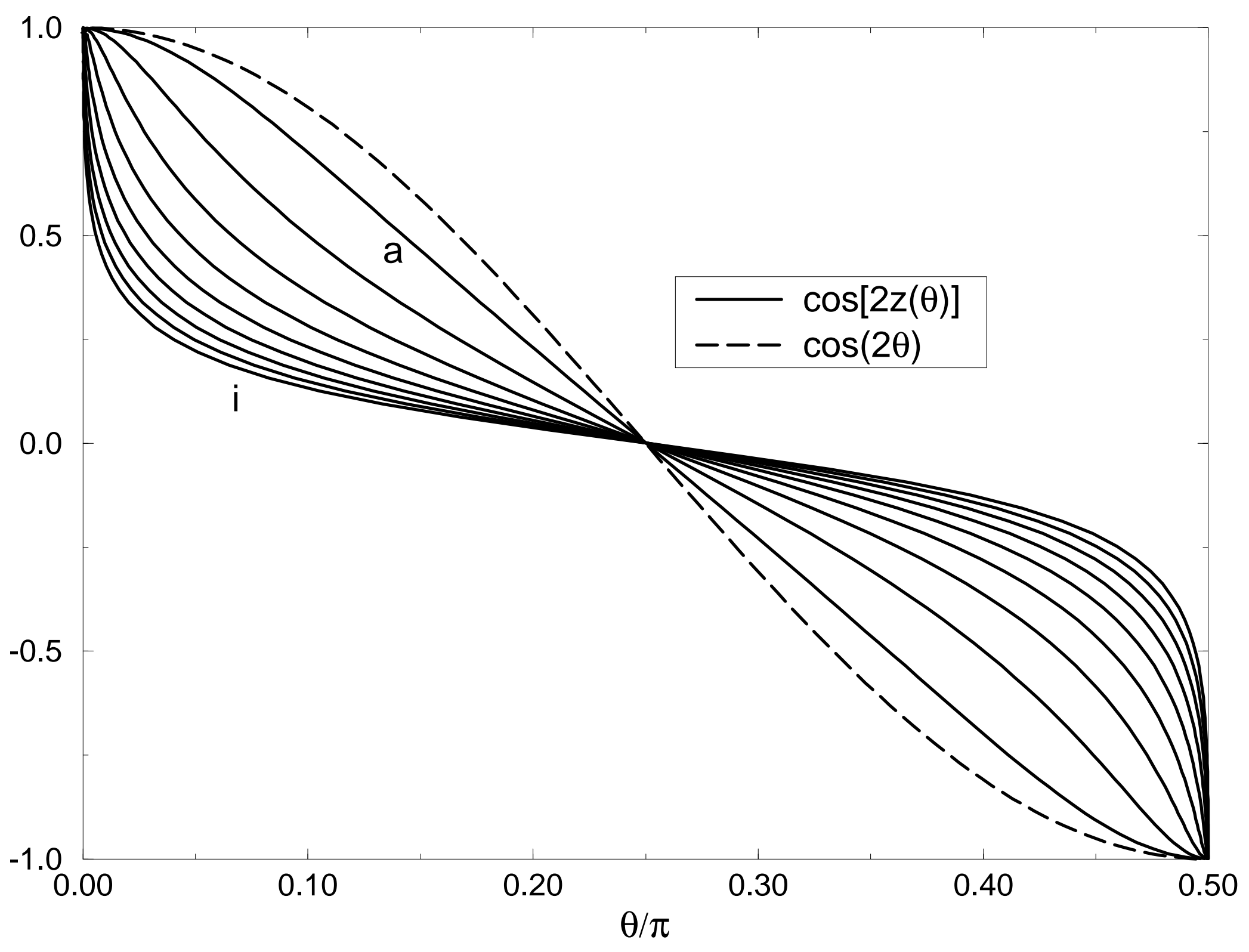




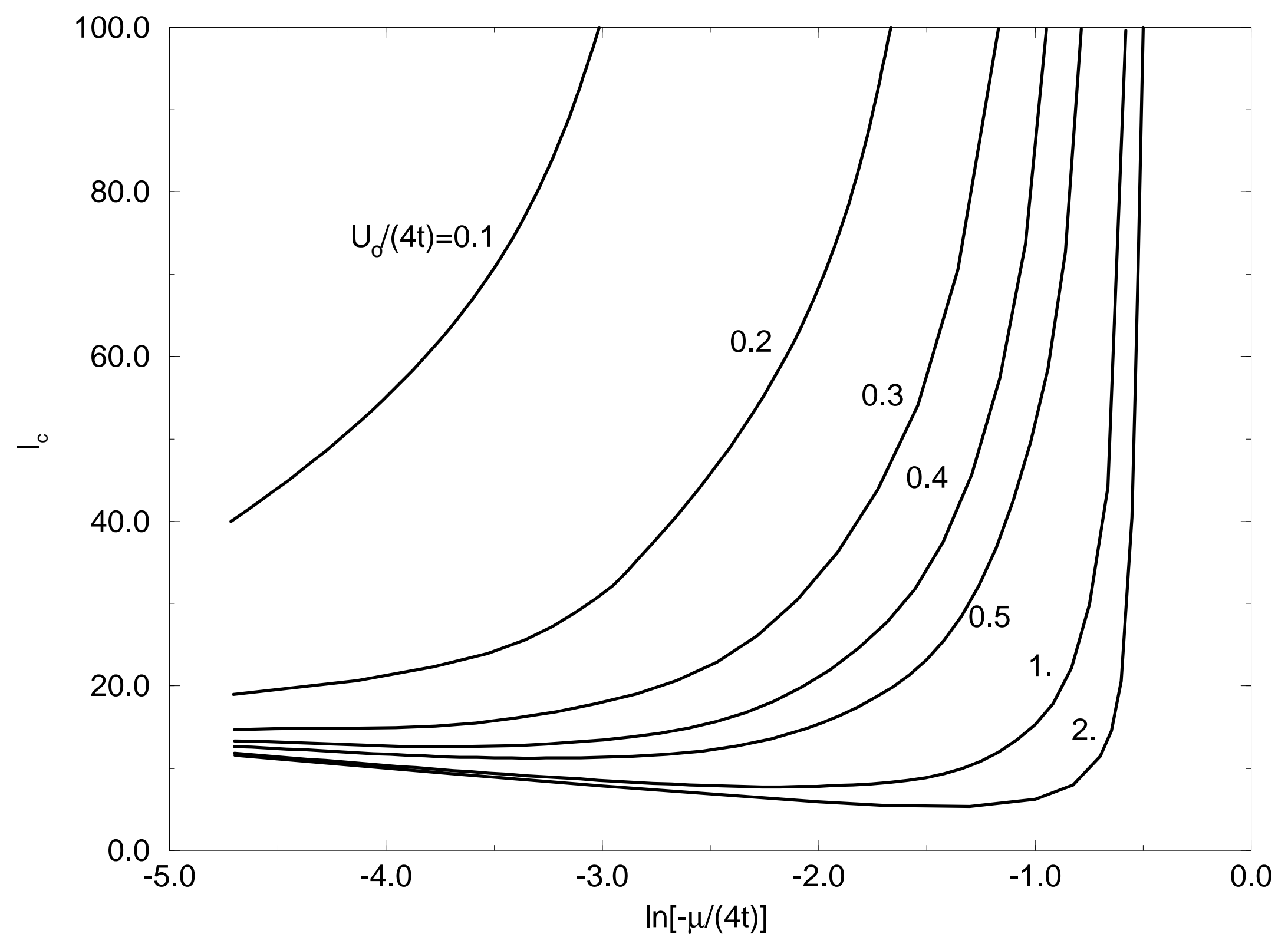

